\title{
13. Conjugate gradient method
}

- conjugate gradient method for linear equations

- complexity

- conjugate gradient method as iterative method

- applications in nonlinear optimization 


\section{Unconstrained quadratic minimization}

$$
\text { minimize } f(x)=\frac{1}{2} x^{T} A x-b^{T} x
$$

with $A$ symmetric positive definite and $n \times n$

- equivalent to solving the linear equation $A x=b$

- the residual $r=b-A x$ is the negative gradient: $r=-\nabla f(x)$

Conjugate gradient method (CG)

- invented by Hestenes and Stiefel around 1951

- the most widely used iterative method for solving $A x=b$, with $A>0$

- can be extended to non-quadratic unconstrained minimization 


\section{Krylov subspaces}

Definition: a sequence of subspaces

$$
\mathcal{K}_{0}=\{0\}, \quad \mathcal{K}_{k}=\operatorname{span}\left\{b, A b, \ldots, A^{k-1} b\right\} \quad \text { for } k \geq 1
$$

\section{Properties}

- subspaces are nested: $\mathcal{K}_{0} \subseteq \mathcal{K}_{1} \subseteq \mathcal{K}_{2} \subseteq \cdots$

- dimensions increase by at most one: $\operatorname{dim} \mathcal{K}_{k+1}-\operatorname{dim} \mathcal{K}_{k}$ is zero or one

- if $\mathcal{K}_{k+1}=\mathcal{K}_{k}$, then $\mathcal{K}_{i}=\mathcal{K}_{k}$ for all $i \geq k$ :

$$
\begin{gathered}
A^{k} b \in \operatorname{span}\left\{b, A b, \ldots, A^{k-1} b\right\} \\
\Downarrow \\
A^{i} b \in \operatorname{span}\left\{b, A b, \ldots, A^{k-1} b\right\} \quad \text { for } i>k
\end{gathered}
$$




\section{Solution of $A x=b$}

\section{Key property:}

$$
A^{-1} b \in \mathcal{K}_{n}
$$

this holds even when $\mathcal{K}_{n} \neq \mathbf{R}^{n}$

- from Cayley-Hamilton theorem,

$$
p(A)=A^{n}+a_{1} A^{n-1}+\cdots+a_{n} I=0
$$

where $p(\lambda)=\operatorname{det}(\lambda I-A)=\lambda^{n}+a_{1} \lambda^{n-1}+\cdots+a_{n-1} \lambda+a_{n}$

- multiplying on the right with $A^{-1} b$ shows

$$
A^{-1} b=-\frac{1}{a_{n}}\left(A^{n-1} b+a_{1} A^{n-2} b+\cdots+a_{n-1} b\right)
$$




\section{Krylov sequence}

\section{Definition}

$$
x_{k}=\underset{x \in \mathcal{K}_{k}}{\operatorname{argmin}} f(x), \quad k=0,1, \ldots
$$

- from previous page, $x_{n}=A^{-1} b$

- CG method is a recursive method for computing the Krylov sequence $x_{0}, x_{1}, \ldots$

- we will see there is a simple two-term recurrence

$$
x_{k+1}=x_{k}-t_{k} \nabla f\left(x_{k}\right)+s_{k}\left(x_{k}-x_{k-1}\right)
$$

\section{Example}

$A=\left[\begin{array}{cc}1 & 0 \\ 0 & 10\end{array}\right], \quad b=\left[\begin{array}{l}10 \\ 10\end{array}\right]$

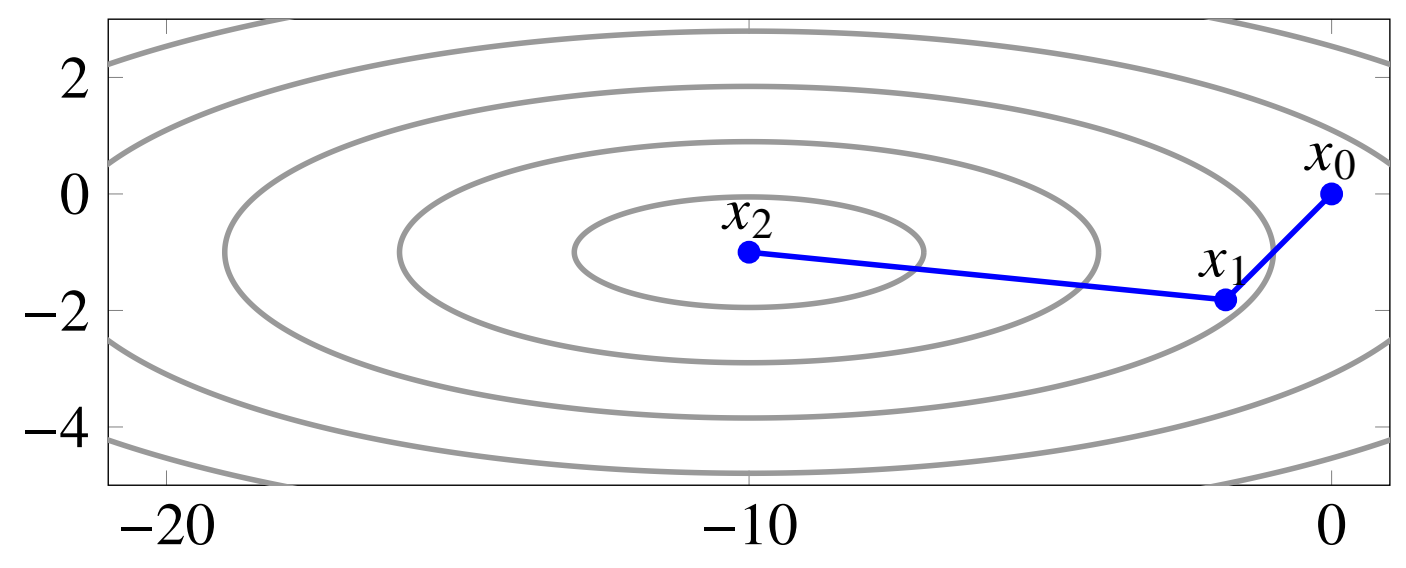




\section{Residuals of Krylov sequence}

$$
x_{k}=\underset{x \in \mathcal{K}_{k}}{\operatorname{argmin}} f(x), \quad k=0,1, \ldots
$$

- optimality conditions in definition of Krylov sequence:

$$
x_{k} \in \mathcal{K}_{k}, \quad \nabla f\left(x_{k}\right)=A x_{k}-b \in \mathcal{K}_{k}^{\perp}
$$

- hence, the residual $r_{k}=b-A x_{k}$ satisfies

$$
r_{k} \in \mathcal{K}_{k+1}, \quad r_{k} \in \mathcal{K}_{k}^{\perp}
$$

the first property follows from $b \in \mathcal{K}_{1}$ and $x_{k} \in \mathcal{K}_{k}$

the (nonzero) residuals form an orthogonal basis for the Krylov subspaces:

$$
\mathcal{K}_{k}=\operatorname{span}\left\{r_{0}, r_{1}, \ldots, r_{k-1}\right\} \quad \text { for } k \geq 1, \quad r_{i}^{T} r_{j}=0 \quad \text { for } i \neq j
$$




\section{Conjugate directions}

the "steps" $v_{i}=x_{i}-x_{i-1}$ in the Krylov sequence (defined for $i \geq 1$ ) satisfy

$$
v_{i}^{T} A v_{j}=0 \quad \text { for } i \neq j, \quad v_{i}^{T} A v_{i}=v_{i}^{T} r_{i-1}
$$

(proof on next page)

- the vectors $v_{i}$ are conjugate: orthogonal for inner product $\langle v, w\rangle=v^{T} A w$

- in particular, if $v_{i} \neq 0$, it is linearly independent of $v_{1}, \ldots, v_{i-1}$

the (nonzero) vectors $v_{i}$ form a conjugate basis for the Krylov subspaces:

$$
\mathcal{K}_{k}=\operatorname{span}\left\{v_{1}, v_{2}, \ldots, v_{k}\right\} \quad \text { for } k \geq 1, \quad v_{i}^{T} A v_{j}=0 \quad \text { for } i \neq j
$$


- assume $j<i$; we show that $A v_{i}$ and $v_{j}$ are orthogonal $\left(v_{i}^{T} A v_{j}=0\right)$ :

$$
v_{j}=x_{j}-x_{j-1} \in \mathcal{K}_{j} \subseteq \mathcal{K}_{i-1}
$$

and

$$
A v_{i}=A\left(x_{i}-x_{i-1}\right)=-r_{i}+r_{i-1} \in \mathcal{K}_{i-1}^{\perp}
$$

- the expression $v_{i}^{T} A v_{i}=v_{i}^{T} r_{i-1}$ follows from the fact that $t=1$ minimizes

$$
f\left(x_{i-1}+t v_{i}\right)=f\left(x_{i-1}\right)+\frac{1}{2} t^{2}\left(v_{i}^{T} A v_{i}\right)-t\left(v_{i}^{T} r_{i-1}\right),
$$

since $x_{i}=x_{i-1}+v_{i}$ minimizes $f$ over the entire subspace $\mathcal{K}_{i}$ 


\section{Conjugate vectors}

it will be convenient to work with a sequence of scaled vectors $p_{k}=v_{k} / \alpha_{k}$ with

$$
\alpha_{k}=\frac{v_{k}^{T} r_{k-1}}{\left\|r_{k-1}\right\|_{2}^{2}}
$$

- the scaling factor $\alpha_{k}$ was chosen to satisfy

$$
p_{k}^{T} r_{k-1}=\left\|r_{k-1}\right\|_{2}^{2}
$$

- using $v_{k}^{T} A v_{k}=v_{k}^{T} r_{k-1}$ (page 13.7), we can express $\alpha_{k}$ as

$$
\alpha_{k}=\frac{p_{k}^{T} r_{k-1}}{p_{k}^{T} A p_{k}}=\frac{\left\|r_{k-1}\right\|_{2}^{2}}{p_{k}^{T} A p_{k}}
$$

- in this notation, the Krylov sequence and residuals satisfy

$$
x_{k}=x_{k-1}+\alpha_{k} p_{k}, \quad r_{k}=r_{k-1}-\alpha_{k} A p_{k}
$$




\section{Recursion for $p_{k}$}

the vectors $p_{1}, p_{2}, \ldots$, can be computed recursively as $p_{1}=r_{0}$,

$$
p_{k+1}=r_{k}-\frac{p_{k}^{T} A r_{k}}{p_{k}^{T} A p_{k}} p_{k}, \quad k=1,2, \ldots
$$

(proof on next page)

- this can be further simplified using (1), (2), and $r_{k} \perp r_{k-1}$ :

$$
r_{k}=r_{k-1}-\frac{\left\|r_{k-1}\right\|_{2}^{2}}{p_{k}^{T} A p_{k}} A p_{k} \quad \Longrightarrow \quad\left\|r_{k}\right\|_{2}^{2}=-\frac{r_{k}^{T} A p_{k}}{p_{k}^{T} A p_{k}}\left\|r_{k-1}\right\|_{2}^{2}
$$

- substituting in the recursion for $p_{k+1}$ gives

$$
p_{k+1}=r_{k}+\frac{\left\|r_{k}\right\|_{2}^{2}}{\left\|r_{k-1}\right\|_{2}^{2}} p_{k}, \quad k=1,2, \ldots
$$


Proof of (3): $p_{k+1} \in \mathcal{K}_{k+1}=\operatorname{span}\left\{p_{1}, p_{2}, \ldots, p_{k}, r_{k}\right\}$, so we can express it as

$$
p_{k+1}=\gamma_{1} p_{1}+\cdots+\gamma_{k-1} p_{k-1}+\beta p_{k}+\delta r_{k}
$$

- $\delta=1$ : take inner product with $r_{k}$ and use

$$
r_{k}^{T} p_{k+1}=\left\|r_{k}\right\|_{2}^{2}, \quad r_{k}^{T} p_{1}=\cdots=r_{k}^{T} p_{k}=0 \quad\left(r_{k} \in \mathcal{K}_{k}^{\perp}\right)
$$

- $\gamma_{1}=\cdots=\gamma_{k-1}=0$ : take inner products with $A p_{j}$ for $j \leq k-1$, and use

$$
p_{j}^{T} A p_{i}=0 \quad \text { for } j \neq i, \quad p_{j}^{T} A r_{k}=0
$$

(second equality because $A p_{j} \in \mathcal{K}_{j+1} \subseteq \mathcal{K}_{k}$ and $r_{k} \in \mathcal{K}_{k}^{\perp}$ )

- hence, $p_{k+1}=r_{k}+\beta p_{k}$; inner product with $A p_{k}$ shows that

$$
\beta=-\frac{p_{k}^{T} A r_{k}}{p_{k}^{T} A p_{k}}
$$




\section{Conjugate gradient algorithm}

define $x_{0}=0, r_{0}=b$, and repeat for $k=0,1, \ldots$ until $r_{k}$ is sufficiently small:

1. if $k=0$, take $p_{1}=r_{0}$; otherwise, take

$$
p_{k+1}=r_{k}+\frac{\left\|r_{k}\right\|_{2}^{2}}{\left\|r_{k-1}\right\|_{2}^{2}} p_{k}
$$

2. compute

$$
\alpha=\frac{\left\|r_{k}\right\|_{2}^{2}}{p_{k+1}^{T} A p_{k+1}}, \quad x_{k+1}=x_{k}+\alpha p_{k+1}, \quad r_{k+1}=r_{k}-\alpha A p_{k+1}
$$

main computation per iteration is matrix-vector product $A p_{k+1}$ 


\section{Outline}

- conjugate gradient method for linear equations

- complexity

- conjugate gradient method as iterative method

- applications in nonlinear optimization 


\section{Notation}

$$
\text { minimize } f(x)=\frac{1}{2} x^{T} A x-b^{T} x
$$

Solution and optimal value

$$
x^{\star}=A^{-1} b, \quad f^{\star}=-\frac{1}{2} b^{T} A^{-1} b=-\frac{1}{2}\left\|x^{\star}\right\|_{A}^{2}
$$

Suboptimality at $x$

$$
f(x)-f^{\star}=\frac{1}{2}\left\|x-x^{\star}\right\|_{A}^{2}
$$

Relative error measure

$$
\tau=\frac{f(x)-f^{\star}}{f(0)-f^{\star}}=\frac{\left\|x-x^{\star}\right\|_{A}^{2}}{\left\|x^{\star}\right\|_{A}^{2}}
$$

here, $\|u\|_{A}=\left(u^{T} A u\right)^{1 / 2}$ is $A$-weighted norm 


\section{Error after $k$ steps}

- $x_{k} \in \mathcal{K}_{k}=\operatorname{span}\left\{b, A b, \ldots, A^{k-1} b\right\}$, so $x_{k}$ can be expressed as

$$
x_{k}=\sum_{i=1}^{k} c_{i} A^{i-1} b=p(A) b
$$

where $p(\lambda)=\sum_{i=1}^{k} c_{i} \lambda^{i-1}$ is a polynomial of degree $k-1$ or less

- $x_{k}$ minimizes $f(x)$ over $\mathcal{K}_{k}$; hence

$$
f\left(x_{k}\right)-f^{\star}=\inf _{x \in \mathcal{K}_{k}} \frac{1}{2}\left\|x-x^{\star}\right\|_{A}^{2}=\inf _{\operatorname{deg} p<k} \frac{1}{2}\left\|\left(p(A)-A^{-1}\right) b\right\|_{A}^{2}
$$

we now use the eigendecomposition of $A$ to bound this quantity 


\section{Error and spectrum of $A$}

- eigendecomposition of $A$

$$
A=Q \Lambda Q^{T}=\sum_{i=1}^{n} \lambda_{i} q_{i} q_{i}^{T} \quad\left(Q^{T} Q=I, \quad \Lambda=\operatorname{diag}\left(\lambda_{1}, \ldots, \lambda_{n}\right)\right)
$$

- define $d=Q^{T} b$

the expression on the previous page simplifies to

$$
\begin{aligned}
2\left(f\left(x_{k}\right)-f^{\star}\right) & =\inf _{\operatorname{deg} p<k}\left\|\left(p(A)-A^{-1}\right) b\right\|_{A}^{2} \\
& =\inf _{\operatorname{deg} p<k}\left\|\left(p(\Lambda)-\Lambda^{-1}\right) d\right\|_{\Lambda}^{2} \\
& =\inf _{\operatorname{deg} p<k} \sum_{i=1}^{n} \frac{\left(\lambda_{i} p\left(\lambda_{i}\right)-1\right)^{2} d_{i}^{2}}{\lambda_{i}} \\
& =\inf _{\operatorname{deg} q \leq k, q(0)=1} \sum_{i=1}^{n} \frac{q\left(\lambda_{i}\right)^{2} d_{i}^{2}}{\lambda_{i}}
\end{aligned}
$$




\section{Error bounds}

\section{Absolute error}

$$
\begin{aligned}
f\left(x_{k}\right)-f^{\star} & \leq\left(\sum_{i=1}^{n} \frac{d_{i}^{2}}{2 \lambda_{i}}\right) \inf _{\operatorname{deg} q \leq k, q(0)=1} \max _{i=1, \ldots, n} q\left(\lambda_{i}\right)^{2} \\
& =\frac{1}{2}\left\|x^{\star}\right\|_{A}^{2} \inf _{\operatorname{deg} q \leq k, q(0)=1} \max _{i=1, \ldots, n} q\left(\lambda_{i}\right)^{2}
\end{aligned}
$$

the equality follows from $\sum_{i} d_{i}^{2} / \lambda_{i}=b^{T} A^{-1} b=\left\|x^{\star}\right\|_{A}^{2}$

\section{Relative error}

$$
\tau_{k}=\frac{\left\|x_{k}-x^{\star}\right\|_{A}^{2}}{\left\|x^{\star}\right\|_{A}^{2}} \leq \inf _{\operatorname{deg} q \leq k, q(0)=1} \max _{i=1, \ldots, n} q\left(\lambda_{i}\right)^{2}
$$




\section{Convergence rate and spectrum of $A$}

- if $A$ has $m$ distinct eigenvalues $\gamma_{1}, \ldots, \gamma_{m}, \mathrm{CG}$ terminates in $m$ steps:

$$
q(\lambda)=\frac{(-1)^{m}}{\gamma_{1} \cdots \gamma_{m}}\left(\lambda-\gamma_{1}\right) \cdots\left(\lambda-\gamma_{m}\right)
$$

satisfies $\operatorname{deg} q=m, q(0)=1, q\left(\lambda_{1}\right)=\cdots=q\left(\lambda_{n}\right)=0$; therefore $\tau_{m}=0$

- if eigenvalues are clustered in $m$ groups, then $\tau_{m}$ is small can find $q(\lambda)$ of degree $m$, with $q(0)=1$, that is small on spectrum

- if $x^{\star}$ is a linear combination of $m$ eigenvectors, CG terminates in $m$ steps take $q$ of degree $m$ with $q\left(\lambda_{i}\right)=0$ where $d_{i} \neq 0$; then

$$
\sum_{i=1}^{n} \frac{q\left(\lambda_{i}\right)^{2} d_{i}^{2}}{\lambda_{i}}=0
$$




\section{Other bounds}

we omit the proofs of the following results

- in terms of condition number $\kappa=\lambda_{\max } / \lambda_{\min }$

$$
\tau_{k} \leq 2\left(\frac{\sqrt{\kappa}-1}{\sqrt{\kappa}+1}\right)^{k}
$$

derived by taking for $q$ a Chebyshev polynomial on $\left[\lambda_{\min }, \lambda_{\max }\right]$

- in terms of sorted eigenvalues $\lambda_{1} \geq \lambda_{2} \geq \cdots \geq \lambda_{n}$

$$
\tau_{k} \leq\left(\frac{\lambda_{k}-\lambda_{n}}{\lambda_{k}+\lambda_{n}}\right)^{2}
$$

derived by taking $q$ with roots at $\lambda_{1}, \ldots, \lambda_{k-1}$ and $\left(\lambda_{1}+\lambda_{n}\right) / 2$ 


\section{Outline}

- conjugate gradient method for linear equations

- complexity

- conjugate gradient method as iterative method

- applications in nonlinear optimization 


\section{Conjugate gradient method as iterative method}

In exact arithmetic

- CG was originally proposed as a direct (non-iterative) method

- in theory, terminates in at most $n$ steps

\section{In practice}

- due to rounding errors, CG method can take many more than $n$ steps (or fail)

- $C G$ is now used as an iterative method

- with luck (good spectrum of $A$ ), good approximation in small number of steps

- attractive if matrix-vector products are inexpensive 


\section{Preconditioning}

- make change of variables $y=B x$ with $B$ nonsingular, and apply $C G$ to

$$
B^{-T} A B^{-1} y=B^{-T} b
$$

- if spectrum of $B^{-T} A B^{-1}$ is clustered, preconditioned CG converges fast

- trade-off between enhanced convergence, cost of extra computation

- the matrix $C=B^{T} B$ is called the preconditioner

\section{Examples}

- diagonal $C=\operatorname{diag}\left(A_{11}, A_{22}, \ldots, A_{n n}\right)$

- incomplete or approximate Cholesky factorization of $A$

- good preconditioners are often application-dependent 


\section{Naive implementation}

apply algorithm of page 13.12 to $\tilde{A} y=\tilde{b}$ where $\tilde{A}=B^{-T} A B^{-1}$ and $\tilde{b}=B^{-T} b$

\section{Algorithm}

define $y_{0}=0, \tilde{r}_{0}=\tilde{b}$, and repeat for $k=0,1, \ldots$ until $\tilde{r}_{k}$ is sufficiently small:

1. if $k=0$, take $\tilde{p}_{1}=\tilde{r}_{0}$; otherwise, take

$$
\tilde{p}_{k+1}=\tilde{r}_{k}+\frac{\left\|\tilde{r}_{k}\right\|_{2}^{2}}{\left\|\tilde{r}_{k-1}\right\|_{2}^{2}} \tilde{p}_{k}
$$

2. compute

$$
\alpha=\frac{\left\|\tilde{r}_{k}\right\|_{2}^{2}}{\tilde{p}_{k+1}^{T} \tilde{A} \tilde{p}_{k+1}}, \quad y_{k+1}=y_{k}+\alpha \tilde{p}_{k+1}, \quad \tilde{r}_{k+1}=\tilde{r}_{k}-\alpha \tilde{A} \tilde{p}_{k+1}
$$




\section{Improvements}

- instead of $y_{k}, \tilde{p}_{k}$ compute iterates and steps in original coordinates

$$
x_{k}=B^{-1} y_{k}, \quad p_{k}=B^{-1} \tilde{p}_{k}
$$

- compute residuals in original coordinates:

$$
r_{k}=B^{T} \tilde{r}_{k}=b-A x_{k}
$$

- compute squared residual norms as

$$
\left\|\tilde{r}_{k}\right\|_{2}^{2}=r_{k}^{T} C^{-1} r_{k}
$$

- extra work per iteration is solving one equation to compute $C^{-1} r_{k}$ 


\section{Preconditioned conjugate gradient algorithm}

define $x_{0}=0, r_{0}=b$, and repeat for $k=0,1, \ldots$ until $r_{k}$ is sufficiently small:

1. solve the equation $C s_{k}=r_{k}$

2. if $k=0$, take $p_{1}=s_{0}$; otherwise, take

$$
p_{k+1}=s_{k}+\frac{r_{k}^{T} s_{k}}{r_{k-1}^{T} s_{k-1}} p_{k}
$$

3. compute

$$
\alpha=\frac{r_{k}^{T} s_{k}}{p_{k+1}^{T} A p_{k+1}}, \quad x_{k+1}=x_{k}+\alpha p_{k+1}, \quad r_{k+1}=r_{k}-\alpha A p_{k+1}
$$




\section{Outline}

- conjugate gradient method for linear equations

- complexity

- conjugate gradient method as iterative method

- applications in nonlinear optimization 


\section{Applications in optimization}

\section{Inexact and truncated Newton methods}

- use conjugate gradient method to compute (approximate) Newton step

- less reliable than exact Newton methods, but handle very large problems

\section{Nonlinear conjugate gradient methods}

- extend linear CG method to nonquadratic functions

- local convergence similar to linear CG

- limited global convergence theory 


\title{
Nonlinear conjugate gradient
}

\author{
minimize $f(x)$
}

$f$ convex and differentiable

Modifications needed to extend linear CG algorithm of page 13.12

- replace $r_{k}=b-A x_{k}$ with $-\nabla f\left(x_{k}\right)$

- determine step size $\alpha$ by line search 


\section{Fletcher-Reeves CG algorithm}

CG algorithm of page 13.12 modified to minimize non-quadratic convex $f$

\section{Algorithm}

choose $x_{0}$ and repeat for $k=0,1, \ldots$ until $\nabla f\left(x_{k}\right)$ is sufficiently small:

1. if $k=0$, take $p_{1}=-\nabla f\left(x_{0}\right)$; otherwise, take

$$
p_{k+1}=-\nabla f\left(x_{k}\right)+\beta_{k} p_{k} \quad \text { where } \quad \beta_{k}=\frac{\left\|\nabla f\left(x_{k}\right)\right\|_{2}^{2}}{\left\|\nabla f\left(x_{k-1}\right)\right\|_{2}^{2}}
$$

2. update $x_{k+1}=x_{k}+\alpha_{k} p_{k+1}$ where

$$
\alpha_{k}=\underset{\alpha}{\operatorname{argmin}} f\left(x_{k}+\alpha p_{k+1}\right)
$$




\section{Some observations}

\section{Interpretation}

- first iteration is a gradient step

- general update is gradient step with momentum term

$$
x_{k+1}=x_{k}-\alpha_{k} \nabla f\left(x_{k}\right)+\frac{\alpha_{k} \beta_{k}}{\alpha_{k-1}}\left(x_{k}-x_{k-1}\right)
$$

- it is common to restart the algorithm periodically by taking a gradient step

\section{Line search}

- with exact line search, reduces to linear CG for quadratic $f$

- exact line search in computation of $\alpha_{k-1}$ implies that $\nabla f\left(x_{k}\right)^{T} p_{k}=0$

- therefore $p_{k+1}$ is a descent direction at $x_{k}$ :

$$
\begin{aligned}
\nabla f\left(x_{k}\right)^{T} p_{k+1} & =-\left\|\nabla f\left(x_{k}\right)\right\|_{2}^{2}+\beta_{k} \nabla f\left(x_{k}\right)^{T} p_{k} \\
& =-\left\|\nabla f\left(x_{k}\right)\right\|_{2}^{2} \\
& <0
\end{aligned}
$$




\section{Variations}

Polak-Ribière: compute $\beta_{k}$ from

$$
\beta_{k}=\frac{\nabla f\left(x_{k}\right)^{T}\left(\nabla f\left(x_{k}\right)-\nabla f\left(x_{k-1}\right)\right)}{\left\|\nabla f\left(x_{k-1}\right)\right\|_{2}^{2}}
$$

Hestenes-Stiefel: compute $\beta_{k}$ from

$$
\beta_{k}=\frac{\nabla f\left(x_{k}\right)^{T}\left(\nabla f\left(x_{k}\right)-\nabla f\left(x_{k-1}\right)\right)}{p_{k}^{T}\left(\nabla f\left(x_{k}\right)-\nabla f\left(x_{k-1}\right)\right)}
$$

formulas are equivalent for quadratic $f$ and exact line search 


\section{References}

- S. Boyd, Lecture slides and notes for EE364b, Convex Optimization II, lectures on the conjugate gradient method.

- G. H. Golub and C. F. Van Loan, Matrix Computations (1996), chapter 10.

- C. T. Kelley, Iterative Methods for Linear and Nonlinear Equations (1995), chapter 2.

- J. Nocedal and S. J. Wright, Numerical Optimization (2006), chapter 5.

- H. A. van der Vorst, Iterative Krylov Methods for Large Linear Systems (2003). 